\title{
A NOVEL TEST OF THE SURGICAL MARGIN IN PATIENTS WITH LUNG CANCER UNDERGOING LIMITED SURGERY: LAVAGE CYTOLOGIC TECHNIQUE
}

\author{
Masahiko Higashiyama, MD, ${ }^{a}$ Ken Kodama, MD, ${ }^{a}$ Hideoki Yokouchi, MD, ${ }^{a}$ Koji Takami, MD, ${ }^{\text {a }}$ Tomio Nakayama, MD, \\ and Takeshi Horai, MD, ${ }^{\mathrm{b}}$ Osaka, Japan
}

\begin{abstract}
Along with the recent increase in the incidence of smallsized lung carcinoma has come the need to perform limited surgery such as segmentectomy, wedge resection under open thoracotomy, or video-assisted thoracic surgery. One of the unfavorable recurrent patterns after such operations is local failure, especially at the surgical margin in the pulmonary parenchyma. Of course, limited surgery should be performed while maintaining safe surgical margins. A tumor-free surgical margin is usually checked macroscopically and, if necessary, by frozen histologic analysis. However, these checking systems appear to be unsatisfactory because the whole surgical margin cannot be examined.

Therefore, we developed a novel checking test, namely "the lavage cytologic method" of examining the surgical margin in patients with lung cancer undergoing limited surgery. In this study, this technique and its preliminary results are shown.
\end{abstract}

Patients and methods. Between October 1997 and March 1999 , limited surgery was performed in 55 consecutive patients with 59 lung cancer lesions in our institute. The patients, aged 34 to 84 years (mean 63.4 years), included 27 men and 28 women. Histologically, 49 lesions were adenocarcinomas, 7 were squamous cell carcinomas, and 3 were large cell carcinomas. Tumor size was from 0.5 to $5.5 \mathrm{~cm}$ (median size $1.9 \mathrm{~cm}$ ). Thirty-two lesions were $2 \mathrm{~cm}$ or smaller, 18 were between 2.1 and $3.0 \mathrm{~cm}$, and 9 were larger than $3.0 \mathrm{~cm}$. Forty-six lesions were stage IA, 11 stage IB, 1 stage IIB, and 1 stage IIIB.

Limited surgery was attempted in 2 groups, designated intentional and compromised. The former group, which included 33 lesions, underwent limited surgery under the criteria of peripheral small-sized stage I lung cancer as described previously by Kodama and associates. ${ }^{1,2}$ The latter group, which included 26 lesions, principally underwent limited resection because of preoperative high-risk complications, multiple lung cancers, or very advanced age.

From the Departments of Thoracic Surgery a and Respiratory Medicine, ${ }^{\mathrm{b}}$ Osaka Medical Center for Cancer and Cardiovascular Diseases, Higashinariku, Osaka, Japan.

Received for publication Feb 24, 2000; accepted for publication March 15, 2000.

Address for reprints: Masahiko Higashiyama, MD, Department of Thoracic Surgery, Osaka Medical Center for Cancer and Cardiovascular Diseases, Nakamichi 1-3-3, Higashinariku, Osaka 537-8511, Japan (E-mail: higamasa@ rj8.so-net.ne.jp).

J Thorac Cardiovasc Surg 2000;120;412-3

Copyright (c) 2000 by The American Association for Thoracic Surgery

$0022-5223 / 2000 \$ 12.00+0 \quad \mathbf{1 2 / 5 4 / 1 0 7 3 4 3}$

doi:10.1067/mtc.2000.107343
For 57 lesions, limited surgery was performed through a thoracotomy, whereas 2 lesions were resected by means of video-assisted thoracoscopic surgery. The operative techniques of wedge resection $(n=24)$ or segmentectomy $(n=33)$ were previously described. A stapler, Nd:YAG laser, electric scissors, or a combination of these devices was used to cut into the pulmonary parenchyma and leave a macroscopically safe margin. In all cases, a pleural lavage cytologic procedure was performed immediately after thoracotomy to avoid tumor cell contamination of the surgical margin. ${ }^{3}$

The lavage cytologic technique of the surgical margin was performed as follows. When limited surgery was performed with a stapler alone, all fired cartridges were washed in 200 $\mathrm{mL}$ of saline solution (Fig 1, $A$ ). When the tumor was excised with the aid of the Nd:YAG laser or electric scissors, alone or in combination with the stapler, the resected specimens were similarly washed without flooding of the pleural surface (Fig $1, B)$. When tumors were resected by means of combined methods, both the used cartridges and the resected samples were washed. These lavage techniques were carefully performed before cross-sections of the specimens were made.

After centrifugation, the sediment was immediately fixed with Saccomanno solution and was then smeared on a glass slide by the cytospin method. Next, after final refixation with ethanol and diethyl ether, the sediment was stained by means of the Papanicolaou method. The cytologic results were judged as positive or negative by a cytologist and were then immediately reported in the operating room. These lavage cytologic examinations usually took approximately 15 minutes to complete.

Results. Four patients with 5 lesions (8\%) showed cytologically positive results in the surgical margin despite a macroscopically safe margin during the attempted operation. Therefore, 1 patient in the intentional group additionally underwent completion lobectomy. In the other patients, who were in the compromised group, no additional resection was performed because of their compromised status and because multiple cancerous lung tumors precluded curative resection. By December 1999, one patient in the compromised group had 2 lesions recur in the surgical margin, whereas no local recurrence was observed in the surgical margin among the patients with cytologically negative results.

Comments. This novel technique for checking for residual tumor cells in the surgical margin offers some advantages over the conventional methods: During the operation, the whole area of the resected margin can be collectively examined and, if necessary, each margin can be separately checked in a relatively short time. In addition, any part of the surgical margin obtained by any cutting method-stapler, Nd:YAG 


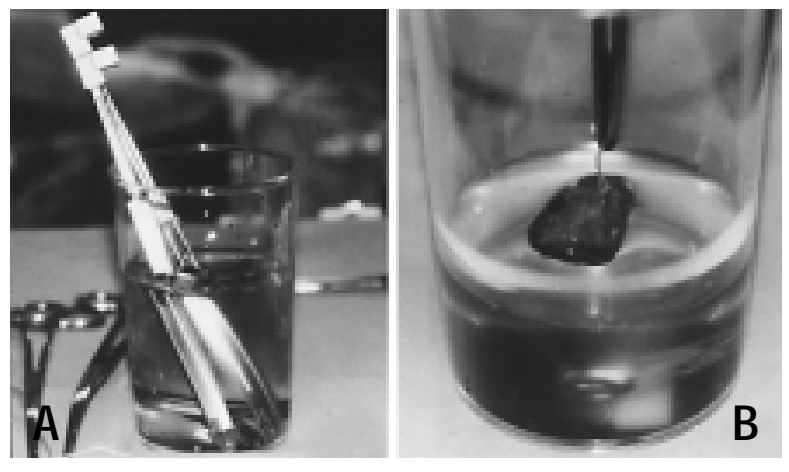

Fig 1. Lavage cytologic technique of the surgical margin in limited surgery for patients with lung cancer. A, When limited surgery was performed with a stapler, its fired cartridge was washed in saline solution for lavage cytology. B, When tumor excision was performed with the $\mathrm{Nd}$ :YAG laser or electric scissors, alone or in combination with the stapler, the resected specimen was washed without flooding of the pleural surface in saline solution for lavage cytology.

laser, or electric scissors - can be examined, whether by open thoracotomy or video-assisted thoracic surgery.

Recently, Sawabata and associates ${ }^{4}$ used a stamped specimen on a glass slide to perform cytologic examination of the surgical margin. This technique, similar to ours, was also useful for detecting tumor cells in the area of the surgical margin. However, we think that our lavage technique may be more complete in estimating the whole area of the surgical margin.

Although the minimum surgical margin for limited surgery at our institution is more than $1 \mathrm{~cm}$, for practical purposes the size of the margin of safety is influenced by anatomic, technical, and histopatholgic conditions. To resect deep-seated tumors or tumors existing in locations where a stapler cannot be anatomically applied, we introduced a promising technique using the Nd:YAG laser. ${ }^{1,2}$ In some instances in these series, however, the surgical margin had to be less than $1 \mathrm{~cm}$; in fact, in 4 of the lesions the margin was cytologically positive despite being macroscopically free of tumor. According to the data of Sawabata and associates, ${ }^{4}$ some patients with a surgical margin of more than $1 \mathrm{~cm}$ also had tumor-positive results. Therefore, this novel check system of the surgical margin may provide practical, useful information in limited surgery. In particular, when a tumor is a histologically smallsized and well-differentiated adenocarcinoma such as a bron- chioloalveolar type, or type IV on Higashiyama's classification, ${ }^{5}$ the tumor itself, as well as its margin, is often undetermined intraoperatively. In such a lesion, the present technique may be even more valuable.

The problem of whether a tumor recurs or not in the cytologically positive surgical margin is clinically interesting. To date, only 1 patient with 2 lesions from the compromised group showed recurrence at the surgical margin, whereas no patient with a cytologically negative surgical margin had a local recurrence, although the follow-up period was short. Therefore, when tumor-positive results after limited operations are obtained by this technique in the intentional group (small peripheral stage I lung cancer), standard resection should be aggressively performed. Even in patients in the compromised group (high-risk complications, multiple lung cancers, or advanced age) who are undergoing limited surgery, additional resection or vaporization of the margin surface of the pulmonary parenchyma with electric scissors or Nd:YAG laser can be selectively performed. Thus, this novel lavage cytologic technique may be a useful test for complete local curability after limited surgery in patients with lung cancer.

We thank Jun-ichi Ashimura, CT, Sachiko Nagumo, CT, and Yasuyoshi Naruse, CT, for their technical assistance in performing cytologic analyses.

\section{REFERENCES}

1. Kodama K, Doi O, Yasuda T, Higashiyama M, Yokouchi H. Radical laser segmentectomy for T1 N0 lung cancer. Ann Thorac Surg 1992:54:1193-5.

2. Kodama K, Doi O, Higashiyama M, Yokouchi H. Intentional limited resection for selected patients with T1 NO M0 non-smallcell lung cancer: a single-institute study. J Thorac Cardiovasc Surg 114;1997:347-53.

3. Higashiyama M, Doi O, Kodama K, Yokouchi H, Tateishi R, Horai T, et al. Pleural lavage cytology immediately after thoracotomy and before closure of the thoracic cavity for lung cancer without pleural effusion and dissemination: clinicopatholgical and prognostic analysis. Ann Surg Oncol 1997;4:409-15.

4. Sawabata N, Mori T, Iuchi K, Maeda H, Ohta M, Kuwahara O. Cytologic examination of surgical margin of excised malignant pulmonary tumor: methods and early results. J Thorac Cardiovasc Surg 1999;117:618-9.

5. Higashiyama M, Kodama K, Yokouchi H, Takami K, Mano M, Kido S, et al. Prognostic value of bronchiolo-alveolar carcinoma component of small lung adenocarcinoma. Ann Thorac Surg 1999;68:2069-73. 